\title{
Effects of gaze stability exercises on cognitive function, dynamic postural ability, balance confidence, and subjective health status in old people with mild cognitive impairment
}

\author{
Miyoung Roh', Eunja Lee,** \\ 'Department of Sports Science, Ewha Womans University, Seoul, Korea \\ ${ }^{2}$ College of Nursing, Gachon University, Incheon, Korea
}

The purpose of this study was to determine whether gaze stability exercises would result in improvements of cognitive function, balance ability and subjective health status in old people with and without mild cognitive impairment (MCI). Old people with $\mathrm{MCl}(n=9)$ and healthy old people $(n=9)$ performed gaze stability exercises for 4 weeks. Pre and post Montreal Cognitive Assessment (MoCA) for cognitive function, Timed Up and Go test for dynamic postural ability, Activities-Specific Balance Confidence for balance confidence and subjective health status were measured in both groups. After participating in gaze stability exercises, all outcome measures were significantly improved in $\mathrm{MCl}$ group and normal group also improved significantly in all outcome measures with the exception of balance confidence. In addition, there were significant differences in cognitive function and balance confidence between the two groups, and more improvements in $\mathrm{MCl}$ group. These results provide evidence that gaze stability exercises is beneficial to improve cognitive function as well as balance ability which affected on quality of life in old people with and without $\mathrm{MCl}$.

Keywords: Gaze stability exercises, Cognitive function, Dynamic postural ability, Balance confidence, Subjective health status, Mild cognitive impairment

\section{INTRODUCTION}

Motor and sensory deteriorations are associated with aging and contribute significantly to the control of dynamic balance (Alexander, 1994; Grabiner and Enoka, 1995). Every year, more than $1 / 3$ of old people experience a fall due to decreased postural stability (Tinetti et al., 1988). Additionally, cognitive functions are impaired with advancing age (Salthouse, 2009) and old people with mild cognitive impairment (MCI) have a higher incidence of falls compared with healthy old people (Liu-Ambrose et al., 2008). As a result, both falls and cognitive impairments can be negatively affected on quality of life of old adults.

Several studies have shown that cognitive and postural impair-

ment are associated with falling (Anstey et al., 2006; Liu-Ambrose et al., 2008). According to Tinetti et al. (1988), old people with cognitive impairment are 5 times greater in odds ratio of falling compared with healthy old people. Many of authors emphasize that we should be aware of MCI as a predictor of falls (Delbaere et al., 2012; Liu-Ambrose et al., 2008). Although there were studies dedicated on cognitive rehabilitation program for patient with cognitive impairment (Kim, 2018; Simon et al., 2012), very little research has focused on fall prevention for old people with MCI. Thus, effective intervention programs may be necessary for preventing falling and dementia in old people with MCI because these people are not only highly at risk of dementia but highly at risk of fall as well (Delbaere et al., 2012).

${ }^{*}$ Corresponding author: Eunja Lee (D https://orcid.org/0000-0003-3525-7829 College of Nursing, Gachon University, 191 Hambakmoe-ro, Yeonsu-gu, Incheon 21936, Korea

E-mail: ejlee@gachon.ac.kr

Received: January 15, 2019 / Accepted: February 8, 2019

This is an Open Access article distributed under the terms of the Creative Commons Attribution Non-Commercial License (http://creativecommons.org/licenses/by-nc/4.0/) which permits unrestricted non-commercial use, distribution, and reproduction in any medium, provided the original work is properly cited. 
Recently, the effects of gaze stability exercises (GSEs) are becoming well known for improving balance of vestibular dysfunction patients in vestibular rehabilitation (Hall et al., 2010; Horning and Gorman, 2007). It has been established for eye movement exercises to improve balance ability and confidence (Park, 2017) and enhance cognitive function (Christman et al., 2003; Parker et al., 2008) respectively in several studies through facilitation of the brain activity and stabilization of one's gaze. However, there is a lack of research on whether GSEs is a beneficial exercise for old people with $\mathrm{MCI}$, not only improving cognitive functions but also ability to balance.

Therefore, the purpose of this study was (a) to investigate the effects of GSEs on cognitive function, balance ability and subjective health status (SHS) in healthy old people with and without MCI and also (b) to determine whether GSEs is more effective for old people with $\mathrm{MCI}$.

\section{MATERIALS AND METHODS}

\section{Participants}

Old people with MCI ( $\mathrm{n}=9,78.9 \pm 5.8$ years) and healthy old people ( $\mathrm{n}=9,74.3 \pm 5.9$ years) participated for this study. They were recruited voluntarily at the Yeonsu Senior Center in Incheon and completed a Montreal Cognitive Assessment (MoCA) to divide into MCI group (18.3 \pm 3.4 ) and normal group (23.8 \pm 1.4$)$. These two groups were matched for age, gender, and education. They had no prior history of neurological diseases and falling that could contribute to balance instability. Informed consent was obtained from the participants prior to participation in this study. Ethics approval was obtained from the Institutional Review Board of Gachon University (1044396-201712-HR-216-01).

\section{GSE intervention}

We conducted GSEs described by Morimoto et al. (2011) and Park (2017). We reconstructed GSEs under the following five steps: (1) eyeball movement, (2) saccadic eye movement, (3) pursuit eye movement, (4) vergence eye movement, (5) vestibular-ocular reflex exercise. Participants were instructed to focus on the card held in their hands with their eyes when moving the target or their head to the left or right in individual's eye level. The eyeball exercise included moving the eyes slowly to the left and right, up and down, rotation movement while closing the eyes. The sac-

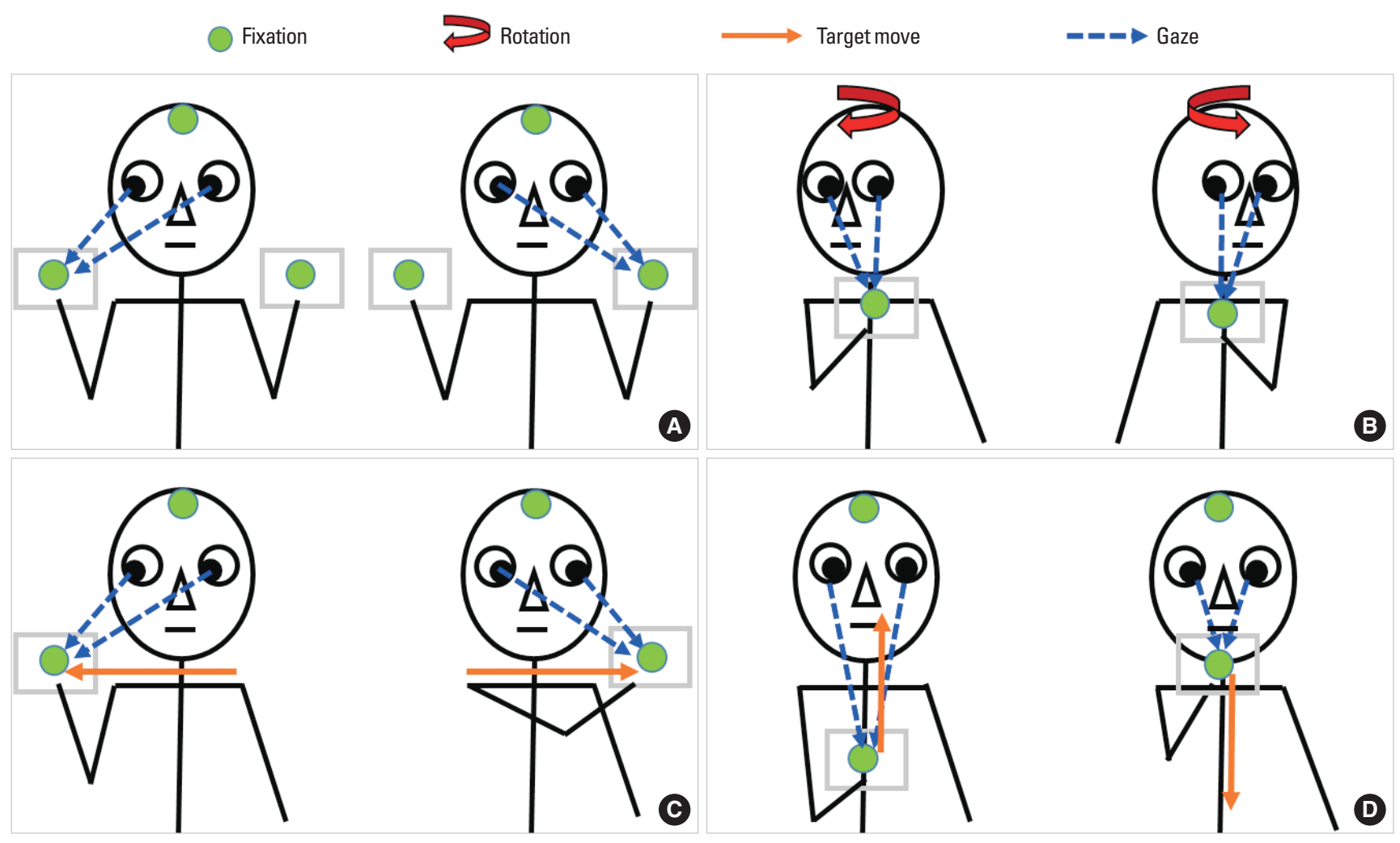

Fig. 1. Four steps of gaze stability exercises illustrations: (A) saccadic eye movement exercise, (B) pursuit eye movement exercise, (C) vestibulo-ocular reflex exercise, and (D) vergence eye movement exercise. 

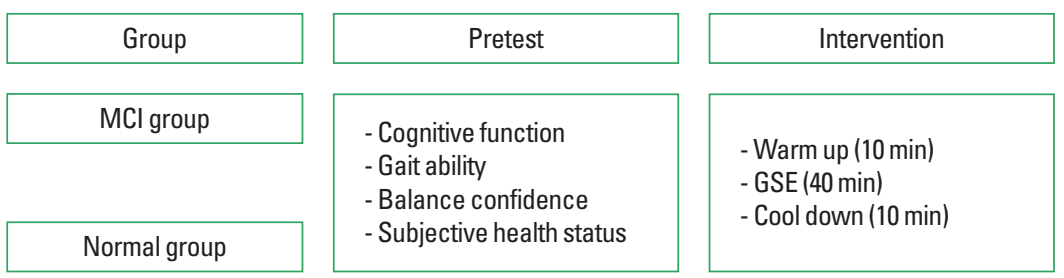

Fig. 2. Process of research. GSE, gaze stability exercise.

cadic eye movement exercise included moving the eyes as quickly as possible between the stationary targets with no head movement. The pursuit eye movement exercise included tracking the slowly moving target holding one hand with the eyes without head movement. The vergence eye movement exercise included tracking the moving target back and forth from $5 \mathrm{~cm}$ close at eye level to far as possible. The vestibular-ocular reflex exercise included moving the head from left to right while keeping the eyes on the stationary target. One hour session of GSEs included a warm up (10 min), GSEs (40 min), and a cool down (10 min). Participants performed GSEs twice a week for 4 weeks in a seated position (Fig. 1).

\section{Outcome measures}

MoCA was used to measure cognitive functions (Nasreddine et al., 2005). MoCA is a 30-point test and evaluates seven areas of cognitive domains: visuospatial ability, executive function, language skill, memory recall, attention, working memory, temporal and spatial orientation. A cut off score of 23 points is used for screening $\mathrm{MCI}$ and a higher score indicates better cognitive function.

Timed Up and Go test was used to assess mobility and balance ability (Podsiadlo and Richardson, 1991). It is a simple test that measured time while subjects rise from a chair, walk for $3 \mathrm{~m}$, turn, walk back and sit down. It has been validated as a predictor of falls (Bischoff et al., 2003; Shumway-Cook et al., 2000). A value lesser than $12 \mathrm{sec}$ is considered high risk of falling in older community-dwelling women.

Activities-specific Balance Confidence was used to measure balance confidence during daily activities such as transferring, bending, and walking (Powell and Myers, 1995). It is a 16-item questionnaire with the scale from 0 (not confidence) to 100 (completely confidence). A higher score indicates better confidence in maintaining balance with the total score $>80 \%$ (high level), $50 \%$ $80 \%$ (medium level), and $<50 \%$ (low level). Cronbach $a$ for this study was 0.97 .

Subjective Health Status was used to measure SHS (Speake et al., 1989). It is a 4-item questionnaire with the scale from 1 (very poor) to 5 (very good). A higher score represents a better health
Table 1. Correlation of variables

\begin{tabular}{lcccc}
\hline Variable & A & B & C & D \\
\hline Cognitive function (A) & 1 & & & \\
Dynamic postural ability (B) & $-0.65^{* *}$ & 1 & & \\
Balance confidence (C) & 0.32 & -0.38 & 1 & \\
Subjective health status (D) & -0.08 & 0.05 & $0.64^{* *}$ & 1 \\
\hline$* * P<0.01$ & & & &
\end{tabular}

status. Cronbach $\alpha$ was 0.87 in this study. All outcome measurements were measured to identify the effects of gaze stability before and after intervention (Fig. 2).

\section{Statistical analysis}

IBM SPSS Statistics ver. 22.0 (IBM Co., Armonk, NY, USA) was used for all analysis. Paired $t$-test was used to evaluate prepost differences of cognitive function, dynamic postural ability, balance confidence and SHS within the group. Independent $t$-test was used to analyze the difference of variables after intervention between groups. The level of statistical significance was accepted at $P<0.05$.

\section{RESULTS}

\section{Correlation between variables}

There was a significantly positive correlation 0.64 between SHS and balance confidence while a negative correlation -0.65 between cognitive function and dynamic postural ability $(P<0.01)$ (Table 1$)$.

\section{Cognitive function}

The paired $t$-test revealed that cognitive function was significantly increased after intervention in both $\mathrm{MCI}$ group $(P<0.01)$ and normal group $(P<0.001)$. The independent $t$-test showed that MCI group had significant increase compared to normal group $(P<0.05)$.

\section{Dynamic postural ability}

The paired $t$-test revealed that dynamic postural ability was sig- 
Table 2. Comparison of variables in $\mathrm{MCl}$ and normal groupsw

\begin{tabular}{|c|c|c|c|c|c|c|}
\hline Variable & Group & Pretest & Posttest & $t^{\text {a) }}$ & Difference & $t^{\mathrm{b})}$ \\
\hline \multirow[t]{2}{*}{ Cognitive function } & $\mathrm{MCl}$ & $18.33 \pm 3.43$ & $23.56 \pm 3.47$ & $-5.31^{* *}$ & $5.22 \pm 2.95$ & $-2.34^{*}$ \\
\hline & Normal & $23.78 \pm 1.39$ & $26.44 \pm 1.74$ & $-5.66^{* * *}$ & $2.67 \pm 1.41$ & \\
\hline \multirow[t]{2}{*}{ Dynamic postural ability } & $\mathrm{MCl}$ & $12.16 \pm 2.17$ & $9.94 \pm 1.62$ & $5.86^{* * *}$ & $-2.21 \pm 1.13$ & $1.76(0.097)$ \\
\hline & Normal & $10.14 \pm 1.73$ & $8.74 \pm 1.35$ & $5.41^{* *}$ & $-1.40 \pm 0.78$ & \\
\hline \multirow[t]{2}{*}{ Balance confidence } & $\mathrm{MCl}$ & $66.74 \pm 21.35$ & $83.82 \pm 13.33$ & $-3.81^{* *}$ & $17.08 \pm 13.45$ & $-2.32^{*}$ \\
\hline & Normal & $83.40 \pm 9.79$ & $87.99 \pm 14.72$ & 1.53 & $4.58 \pm 9.01$ & \\
\hline \multirow[t]{2}{*}{ Subjective health status } & $\mathrm{MCl}$ & $2.86 \pm 0.88$ & $4.22 \pm 0.74$ & $-4.44^{* *}$ & $1.36 \pm 0.92$ & -0.32 \\
\hline & Normal & $3.11 \pm 0.44$ & $4.36 \pm 0.53$ & $-7.75^{* * *}$ & $1.25 \pm 0.48$ & \\
\hline
\end{tabular}

$\mathrm{MCl}$, mild cognitive impairment.

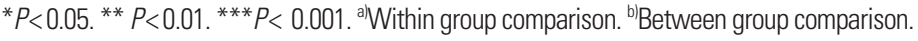

nificantly increased in both $\mathrm{MCI}$ group $(P<0.001)$ and normal group $(P<0.01)$. Whereas dynamic postural ability was increased in both groups after intervention, there was no statistically significant difference between groups by the independent $t$-test (Table 2).

\section{Balance confidence}

The paired $t$-test revealed that balance confidence was significantly increased in MCI group $(P<0.01)$ while no significant difference in normal group. The independent $t$-test showed that MCI group had significant increase compared to normal group $(P<0.05)$ (Table 2).

\section{Subjective health status}

The paired $t$-test revealed that SHS was significantly increased in both MCI group $(P<0.01)$ and normal group $(P<0.001)$. There was no statistically significant difference between groups by the independent $t$-test (Table 2).

\section{DISCUSSION}

There have been many research focused on benefits of cognitive rehabilitation programs for old people with MCI. Recently, MCI was found not only highly at risk of dementia but also as a predictor of falling (Delbaere et al., 2012). However, there has been very little research on fall prevention exercise for old people with MCI. Thus, this study applied and examined the GSE as an effective tool for both cognitive function and balance ability in old people with MCI. Furthermore, we assumed that GSE led to the greater improvement for old people with MCI compared with healthy old people.

To our knowledge, this is the first study to demonstrate the direct effects of GSE on both cognitive function and balance ability in old people with and without MCI. The results of this study in- dicated that $\mathrm{MCI}$ and normal group improved in measures of dynamic postural stability and also cognitive function and SHS after GSEs. In addition, there were significant differences in cognitive function and balance confidence between the two groups, and there were more improvements in $\mathrm{MCI}$ group. These results are consistent with the evidence that eye movement exercises results in significant improvements in postural stability of patients with dizziness (Hall et al., 2010) and fall experience (Park, 2017). However, many studies have examined only the effects of the eye movement exercises on postural stability in vestibular rehabilitation despite of the evidence that cognition and posture are related to each other (Liu-Ambrose et al., 2008).

Unlike the previous studies, we hypothesized about the effects of GSEs for improving both cognitive function and balance in old people with MCI since they are high risk of dementia and falling. In this study, all participants performed GSEs consisted of four different type of bilateral eye movement; saccadic eye movement, pursuit eye movement, vestibular-ocular reflex exercises, and vergence eye movement during 4 weeks. The possible mechanism contributing to balance enhancement would be the adaptation of vestibular (Hall et al., 2010) through vestibule-ocular reflex exercise. This hypothesis goes with vestibular rehabilitation studies (Hall et al., 2010; Morimoto et al., 2011) that vestibular-specific GSEs results in greater improvements of dynamic visual acuity and postural stability by facilitating the interaction of vision and vestibular system related to postural control.

Second possible mechanism leading to cognitive improvement is that bilateral saccadic eye movement, in line with the principle of bilateral movement, enhance interhemispheric interaction (Christman et al., 2003; Parker et al., 2008) related to superior episodic memory by stimulating the left and right hemispheres. This hypothesis is based on great improvements in cognitive functions after GSE although we did not measure the brain activi- 
ty. As far as we know, this is the first study that we confirmed the effects of GSE by directly measuring cognitive functions and balance ability and SHS in old people with MCI.

In conclusion, GSE is beneficial to improve cognitive function as well as balance ability which affected on quality of life in old people with and without MCI. Thus, we should consider providing GSEs, easy, simple and cost-effective home based exercise, that including components with related to improvements of cognitive function and balance in old people with $\mathrm{MCI}$ and having experience of fallings. In this study, the number of participants was limited and we did not measure ocular motor function to identify mechanisms underlying changes in neural adaptation. Further investigations should examine the neural mechanisms of how eye movement exercises promote balance and cognitive function respectively with larger sample size.

\section{CONFLICT OF INTEREST}

No potential conflicts of interest relevant to this study was reported.

\section{ACKNOWLEDGMENTS}

This study was supported by the National Research Foundation of Korea Grant funded by the Korean Government (NRF-2016S1A5B5A07921409).

\section{REFERENCES}

Alexander NB. Postural control in older adults. J Am Geriatr Soc 1994;42: 93-108.

Anstey KJ, von Sanden C, Luszcz MA. An 8-year prospective study of the relationship between cognitive performance and falling in very old adults. J Am Geriatr Soc 2006;54:1169-1176.

Bischoff HA, Stähelin HB, Monsch AU, Iversen MD, Weyh A, von Dechend M, Akos R, Conzelmann M, Dick W, Theiler R. Identifying a cutoff point for normal mobility: a comparison of the timed 'up and go' test in community-dwelling and institutionalised elderly women. Age Ageing 2003;32:315-320.

Christman SD, Garvey KJ, Propper RE, Phaneuf KA. Bilateral eye movements enhance the retrieval of episodic memories. Neuropsychology 2003;17:221-229.

Delbaere K, Kochan NA, Close JC, Menant JC, Sturnieks DL, Brodaty H, Sachdev PS, Lord SR. Mild cognitive impairment as a predictor of falls in community-dwelling older people. Am J Geriatr Psychiatry

\section{2;20:845-853.}

Grabiner MD, Enoka RM. Changes in movement capabilities with aging. Exerc Sport Sci Rev 1995;23:65-104.

Hall CD, Heusel-Gillig L, Tusa RJ, Herdman SJ. Efficacy of gaze stability exercises in older adults with dizziness. J Neurol Phys Ther 2010;34: 64-69.

Horning E, Gorman S. Vestibular rehabilitation decreases fall risk and improves gaze stability for an older individual with unilateral vestibular hypofunction. J Geriatr Phys Ther 2007;30:121-127.

Kim S. Exploring the field application of combined cognitive-motor program with mild cognitive impairment elderly patients. J Exerc Rehabil 2018;14:817-820.

Liu-Ambrose TY, Ashe MC, Graf P, Beattie BL, Khan KM. Increased risk of falling in older community-dwelling women with mild cognitive impairment. Phys Ther 2008;88:1482-1491.

Morimoto H, Asai Y, Johnson EG, Lohman EB, Khoo K, Mizutani Y, Mizutani T. Effect of oculo-motor and gaze stability exercises on postural stability and dynamic visual acuity in healthy young adults. Gait Posture 2011;33:600-603.

Nasreddine ZS, Phillips NA, Bédirian V, Charbonneau S, Whitehead V, Collin I, Cummings JL, Chertkow H. The Montreal Cognitive Assessment, MoCA: a brief screening tool for mild cognitive impairment. J Am Geriatr Soc 2005;53:695-699.

Park JH. The effects of eyeball exercise on balance ability and falls efficacy of the elderly who have experienced a fall: a single-blind, randomized controlled trial. Arch Gerontol Geriatr 2017;68:181-185.

Parker A, Relph S, Dagnall N. Effects of bilateral eye movements on the retrieval of item, associative, and contextual information. Neuropsychology 2008;22:136-145.

Podsiadlo D, Richardson S. The timed "Up \& Go": a test of basic functional mobility for frail elderly persons. J Am Geriatr Soc 1991;39:142-148.

Powell LE, Myers AM. The Activities-specific Balance Confidence (ABC) Scale. J Gerontol A Biol Sci Med Sci 1995;50A:M28-34.

Salthouse TA. When does age-related cognitive decline begin? Neurobiol Aging 2009;30:507-514.

Shumway-Cook A, Brauer S, Woollacott M. Predicting the probability for falls in community-dwelling older adults using the Timed Up \& Go Test. Phys Ther 2000;80:896-903.

Simon SS, Yokomizo JE, Bottino CM. Cognitive intervention in amnestic Mild Cognitive Impairment: a systematic review. Neurosci Biobehav Rev 2012;36:1163-1178.

Speake DL, Cowart ME, Pellet K. Health perceptions and lifestyles of the elderly. Res Nurs Health 1989;12:93-100.

Tinetti ME, Speechley M, Ginter SF. Risk factors for falls among elderly persons living in the community. N Engl J Med 1988;319:1701-1707. 